\title{
PROCRASTINACIÓN ACADÉMICA Y ADICCIÓN A INTERNET EN ESTUDIANTES UNIVERSITARIOS DE LIMA METROPOLITANA
}

\section{Academic procrastination and internet addiction in university students of Lima Metropolitana}

Silvia Castro Bolaños* Karime Mahamud Rodríguez**

\section{Resumen}

Elobjetivo de la presente investigación es determinar la relación entre procrastinación académica y adicción a internet en jóvenes estudiantes de una universidad privada de Lima Metropolitana. La muestra la conforman 168 alumnos de ambos sexos, con edades comprendidas entre 18 y 25 años de una universidad privada de Lima Metropolitana. Los materiales utilizados fueron una escala de procrastinación y una escala de adicción a internet. Los resultados muestran una relación positiva y significativa entre procrastinación académica y adicción a internet.

Palabras clave: Procrastinación académica, adicción a internet, estudiantes universitarios.

\begin{abstract}
The purpose of this investigation is determining the relationship between academic procrastination and internet addiction in young students of a private university in Lima Metropolitana. In the sample were considered 168 students of both genders, aged between 18 and 25 years of a private university in Lima Metropolitana. The materials used were a procrastination scale and an internet addiction scale. The results show a positive and significant relationship between academic procrastination and internet addiction.
\end{abstract}

Keywords: Academic procrastination, internet addiction, university students.

\footnotetext{
* Psicóloga clínica. silviacastroolano@gmail.com

** Psicóloga clínica. karimemahamud@hotmail.com

Artículo basado en la tesis de licenciatura sustentada por las autoras.
} 


\section{INTRODUCCIÓN}

Actualmente las tecnologías de información y el uso de internet es cada vez más utilizado por la población adolescente. Por un lado constituyen una fuente valiosa de información y de interacción social pero, por otro lado, existen algunos riesgos como la dependencia que se pueden suscitar en esta población (Toro, 2010).

Asimismo la procrastinación académica es una realidad que puede llevar a los adolescentes a obtener bajo rendimiento académico y en algunos casos deserción. Diariamente el uso indiscriminado de las tecnologías de información podría volver más fácil el procrastinar. De esta manera, causar efectos perjudiciales para el desarrollo de la población adolescente en el ámbito académico si estos medios no son utilizados adecuadamente.

El objetivo es determinar la relación existente entre la procrastinación académica y la adicción a internet en la población universitaria.

\section{ANTECEDENTES}

\section{Procrastinación Académica}

Se considera procrastinación académica cuando se aplazan las tareas del contexto escolar, tanto académicas o administrativas, pero el procrastinar no es un comportamiento que solo se encuentra en el área académica, sino, en diversas áreas. Esta procrastinación académica, puede estar relacionada con la presencia de comportamientos de aplazamiento en otras áreas (Quant y Sánchez, 2012). De acuerdo a Steel (2007), la procrastinación académica se inicia en la educación secundaria y persiste en la universidad, donde más la mitad de los estudiantes refieren tener retrasos constantes y problemáticos a la hora de realizar sus tareas. Cuando existe un retraso no justificado, es decir, innecesario e irracional, para la ejecución o conclusión de tareas y ocasiona conflicto en el individuo, se llama procrastinación. Se presenta un conflicto entre la intención y la acción, ya que no hay una concordancia entre lo que se desea y se realiza; no existiendo este retraso solo en el área académica sino en diversas áreas y contextos, abarcando diversas situaciones y circunstancias, las cuales se presentan tanto en el inicio como en la finalización de cualquier actividad (Natividad, 2014).
A la procrastinación se le puede considerar como un problema de autocontrol y de organización del tiempo, de tal manera se puede evaluar de qué forma y desde cuando se instaura el hábito de posponer las actividades para más adelante. La procrastinación en los jóvenes afecta, no solo, el tomar decisiones, sino también, a la hora de resolver o de enfrentarse a conflictos, ya sea, en cuanto a terminar sus obligaciones académicas o satisfacer los requerimientos de su entorno. Asimismo, si en la etapa de la adolescencia estos hábitos están instaurados, es decir, las conductas de procrastinación se mantienen, esto no solamente influirá en su desempeño académico, sino, que a largo plazo se verá reflejado en actividades familiares, sociales, laborales entre otras (Chan, 2011).

\section{Procrastinación académica y procrastinación de la vida diaria}

Cuando hay algo que pueda causar molestia, algo con lo que uno no se siente en la capacidad de realizarlo adecuadamente, algo que no lo motive, es decir, no sea significativo en ese momento; lo evita, y a ese comportamiento de evitación y postergación a la ejecución de determinada actividad se le denomina procrastinación. Estas ideas se pueden relacionar con creencias personales tempranas que perduran hasta la adultez, las cuales se vinculan en varias áreas como a la hora de tomar una decisión, resolver problemas, hacer frente a situaciones de estrés que sean motivadas por las tareas y en la organización de actividades para el logro de los objetivos. Por estos motivos, el procrastinador no es capaz de concentrar su atención en actividades importantes, su pensamiento es a corto plazo sin poder planificar más hacia el futuro, y con su conducta busca evitar o minimizar esta sensación, que, a la vez, presenta cierta vulnerabilidad biológica hacia un estado de tensión con alguna impulsividad (Angarita, 2012).

La procrastinación no es dejar de realizar una actividad o tarea, sino posponerla, y en actividades diarias podría generar ansiedad y preocupación pero no necesariamente trae consecuencias negativas ya que finalmente se llegan a cumplir. Sin embargo, en el área académica investigaciones han encontrado que el $25 \%$ de estudiantes tienen la tendencia a posponer o dejar de lado actividades para encargarse posteriormente y esta procrastinación se relaciona con negativas 
consecuencias académicas (Ellis \& Knaus, 1977; Solomon \& Rothblum, 1984; Rothblum, Solomon \& Murakami, 1986, citados por Álvarez. 2010).

\section{Procrastinadores activos y procrastinadores pasivos}

De acuerdo a Hsin y Nam (2005) hay dos tipos de procrastinadores: los procrastinadores activos y los procrastinadores pasivos. Los procrastinadores activos, postergan el trabajo, prefieren trabajar bajo presión y tomar decisiones deliberadas para posponer las cosas. Los procrastinadores pasivos no planean procrastinar, dejan de realizar las actividades en su momento. En ambos casos las personas procrastinan de la misma forma, habiendo características similares tanto en los procrastinadores activos como pasivos que se relacionan con el manejo de tiempo, la percepción de autoeficacia y los estilos de afrontamiento.

Asimismo señalan que entre los procrastinadores activos y los no procrastinadores; los primeros presentan menos estrés obteniendo mejor salud física cuando las fechas de entrega son más a largo plazo, a diferencia de los no procrastinadores. De tal manera, la procrastinación puede ser considerada como una estrategia que ayuda a regular las emociones negativas, obteniendo una sensación temporal de bienestar, a la vez, encontraron que posponer una actividad o tarea para los procrastinadores activos no interfiere o altera la ejecución de sus labores, al igual que el posponer, les genera bienestar temporal. El postergar la actividad, hace que el trabajo lo realice con un buen desempeño y obtengan mejores resultados. Para los procrastinadores pasivos, el postergar las tareas no está dentro de sus planes, lo que se les dificulta es el poder tomar una decisión de una manera eficaz y eficiente, pero, al acercarse la fecha de entrega sienten presión, originándoles pensamientos negativos afectando la percepción en sus capacidades, y por ende, no obteniendo resultados óptimos.

\section{Adicción}

Cuando existe cierta inclinación desmedida hacia alguna actividad puede desembocar en una adicción, ya sea que exista o no una sustancia química de por medio. Se define a la adicción como una afición patológica que genera dependencia y el ser humano pierde su libertad, ya que, lo que ocasiona es un estrechamiento de su campo de conciencia y restringe la amplitud de sus intereses. De hecho, existen hábitos de conducta en apariencia inofensivos que, en determinadas circunstancias, pueden convertirse en adictivos e interferir gravemente en la vida cotidiana de las personas afectadas, a nivel familiar, escolar, social o de salud (Echeburúa y Corral, 1994; citados por Echeburúa y Corral, 2010).

La adicción está caracterizada por la pérdida de control y la dependencia. Al inicio las conductas de adicción están controladas por reforzadores positivos, el aspecto placentero de la conducta, pero en el proceso son controladas por reforzadores negativos, principalmente, el alivio de la tensión emocional. (Marks, 1990; Potenza, 2006; Treuer, Fábian y Füredi, 2001; citados por Echeburúa y Corral 2010).

\section{Internet}

Internet se define como una red de redes de ordenadores que comparten datos y recursos. Existe una conexión de redes a nivel mundial que permite a los ordenadores (y a las personas) comunicarse entre sí a nivel global, pudiendo mantener un acceso hacia la información y personas que de otro modo no sería posible. El internet permite innumerables ventajas; tanto para la educación, el comercio, el entretenimiento y para el desarrollo de la persona (Luengo, 2004).

La interacción en el internet podría conllevar a la desinhibición, por su característica anónima en la interacción, a diferencia de la vida real. El pudor y el miedo quedan disminuidos, siendo posible expresarse de una manera libre, haciendo que la persona se sienta protegida y menos expuesta. Fácilmente, uno puede relacionarse no solo con una, sino, con muchas personas que tengan los mismos intereses. Es una herramienta que ayuda a las personas que se sienten estigmatizadas, ya sea por sexo o ideología, puedan vincularse sin temor, logrando una auto aceptación, mostrándose tal como y realmente quieren ser. Por tal motivo, la permanencia en el grupo virtual se convierte en una parte importante de su identidad (Greenfield, 1999; McKenna y Bargh 1998; citados por Luengo, 2004).

Internet constituye una tecnología que ha generado un impacto primordial en la juventud, brindándoles 
diversos beneficios. Sin embargo, se presentan casos en los cuales algunas personas llegan a estar obsesionadas con internet, mostrando conductas incapaces para controlar su uso y pueden poner en peligro su trabajo y sus relaciones (Echeburúa y Corral, 2010).

\section{Adicción a internet}

En el mundo actual una herramienta que forma parte de la sociedad es el internet, que, inicialmente, permite hacer mejoras diarias como obtener información, mantenerse en contacto, etc. Sin embargo, cuando se da un uso no formal, de forma recreativa, excesiva y persistente, puede llevar a una adicción. Considerándose este fenómeno en el área clínica y epidemiológica como una de las patologías emergentes de mayor impacto en el siglo actual. Para que sea considerada una adicción, la persona tiene un comportamiento en el cual permanece muchas horas durante el día conectado con fines recreativos, descuidando las diferentes áreas de su vida, tales como, el trabajo, amistades, alimentación, descanso, etc.; y manifiestan síntomas de ansiedad, irritabilidad, desajuste emocional y problemas en la interacción social. Se les hace más fácil poder comunicarse a través del internet que la interacción personal, pueden crear a alguien que no sean ellos mismos, como por ejemplo, un personaje idealizado. Pueden pasar muchas horas frente a actividades como el chat, navegar en la web, juegos de azar online, los juegos en red, el relacionamiento social virtual, o la búsqueda compulsiva de sexo, juegos violentos o pornografía (Cía, 2013).

Para que sea considerada una adicción, no solamente se debe tomar en cuenta el uso frecuente del internet, sino la relación que mantiene con este uso, es decir, el grado de dependencia, la falta de control y la desmedida interferencia que genera en su vida diaria. Por lo tanto, es de suma importancia, determinar claramente un estado de dependencia del celular con el interés hacia las nuevas tecnologías, o disfrutar de las redes sociales con tener una adicción.

La adicción a las nuevas tecnologías se considera dentro del ámbito de las adicciones psicológicas, siendo similar a la adicción a las compras, el sexo o los juegos, las cuales generan una fuerte dependencia psicológica, pérdida de control, interferencia en la vida cotidiana y presencia del síndrome de abstinencia (Echeburúa y Amor, 2001; citados por Oliva, et al., 2012).

Existen hábitos de conducta aparentemente inofensivos que, en determinadas circunstancias, pueden convertirse en adictivos e interferir gravemente en la vida cotidiana de las personas afectadas, a nivel familiar, escolar, social o de salud (Echeburúa y Corral, 1994; citados por Echeburúa y Corral, 2010). La persona que desarrolla una adicción a internet, tiene un comportamiento en donde permanece largas horas bajo conexión con fines recreativos a lo largo de cada día, lo que le genera el descuido en diversas áreas importantes de su vida tales como el trabajo, las relaciones sociales, la alimentación, el descanso, etc. Los adictos a internet comienzan a manifestar síntomas ansiosos, irritabilidad, desajuste emocional y problemas en la interacción social (Cía, 2013).

\section{Adolescencia y adicción a internet}

El periodo de la adolescencia es considerado como un fenómeno psicológico, biológico y cultural en donde el adolescente se desarrolla adquiriendo una madurez psicológica construyendo una identidad personal, y posee características propias en las cuales se denota omnipotencia, tendencia a buscar la causa de sus problemas en el otro, inexperiencia de vida, dificultad de reconocer adicciones sutiles y necesidad de normalizar conductas de riesgo; de tal manera, convierte a este periodo en una época susceptible a sufrir conductas adictivas u otros trastornos psicológicos relacionados con el uso de algunas aplicaciones de las tecnologías de información (Castellana, 2003, 2005, citada por Castellana, Sánchez-Carbonell, Graner y Beranuy, 2007)

El uso del internet de manera excesiva y sin control puede generar una conducta adictiva, que lo lleva a una dedicación excesiva, apartando a la persona de sus actividades de la vida diaria, desarrollando una conducta de dependencia y sin control. El adolescente es más proclive a esta tipo de adicción que el adulto, debido a que su corteza prefrontral se encuentra aún inmadura y su autocontrol no ha alcanzado aún el nivel adulto (Oliva, et al., 2012). Cuando se habla de adolescentes en donde el uso de la tecnología no es utilizado como un medio, sino, como un fin, se habla 
de conductas adictivas. Dichas conductas lo llevan a sentir un gran deseo por obtener el móvil de última generación o quedar atrapado en las redes sociales, creando y disfrutando de una identidad falsa e irreal (Becoña, 2006, citado por Echeburúa y Corral, 2010).

La mayor parte de investigaciones y estudios de adicción a internet en estudiantes se han realizado en los países de Taiwán, Corea del Sur y China; en donde han hallado que la incidencia de adicción a internet durante un año era del 7,5 por 100, y la tasa de remisiones, también durante un año, del 45,9 por 100 (Ko, Yen, Yen, et. al., citados por Toro, 2010). Asimismo, la adicción a internet en Corea del Sur es considerado uno de los principales problemas de salud pública, señalando en un estudio que el 2,1 por 100 de la población de 6 a 19 años de edad, sufrían de adicción a las nuevas tecnologías y requerían tratamiento; un $80 \%$ requerían de medicación psicotrópica y del 20 al $24 \%$ debían ser hospitalizados (Block, citado por Toro, 2010).

\section{MÉTODO}

\section{Diseño}

Investigación cuantitativa y de tipo correlacional.

\section{Participantes}

Los participantes de la investigación fueron 168 estudiantes desde el segundo hasta el noveno ciclo de una universidad privada de Lima Metropolitana, 97 hombres y 71 mujeres. El rango de edad de la población encuestada fluctuaba entre 18 y 25 años. La edad promedio fue de 19 años y 6 meses. Siendo la mayor cantidad de alumnos con una edad de 18 años representando $37.5 \%(n=63)$ de la población encuestada y el $20.8 \%(\mathrm{n}=35)$ tiene 19 años.

\section{Instrumentos}

\section{Escala de Procrastinación}

Parala variableprocrastinaciónacadémicaseutilizó una Escala de Procrastinación, compuesta por 12 ítems y se mide utilizando una escala en formato Likert. Esta escala evalúa dos factores: Autorregulación, entendida como un proceso activo en el que los estudiantes establecen sus objetivos principales de aprendizaje y a lo largo de este, tratan de conocer, controlar y regular sus cogniciones, motivaciones y comportamientos de cara a alcanzar esos; y, Postergación, comprende las acciones de aplazar las actividades académicas.

\section{Escala de Adicción a Internet}

Para la variable de adicción a internet se utilizó una Escala de Adicción a Internet. La escala está compuesta por 11 ítems y cada uno cuenta con respuestas de opción múltiple en formato Likert. La presente escala evalúa dos dimensiones: sintomatología: Saliencia (estar preocupado por la utilización de internet), Tolerancia, abstinencia, falla en el control y recaída. Cuenta con 8 ítems para evaluar esta dimensión, y la segunda dimensión; características disfuncionales: problemas académicos, familiares e interpersonales. Cuenta con 3 ítems para evaluar esta dimensión. La confiabilidad fue estimada mediante el Alfa de Cronbach resultando para la escala total .84. El análisis bidimensional mostró asociación significativa $(\mathrm{p}<0,001)$ entre la dimensión I (Sintomatología de AI) y el tiempo de uso semanal, sexo masculino, antecedente de problemas de indisciplina y plan de futuro. La dimensión II (Disfuncionalidad por AI) se asoció significativamente $(p<0,001)$ al antecedente de problemas de indisciplina, plan de futuro y faltar sin motivo justificable a la escuela.

\section{Procedimiento}

Para efectos de la presente investigación, se procedió a aplicar una Escala de Procrastinación a 168 estudiantes de una universidad privada de Lima Metropolitana. Posteriormente se tabularon los resultados y fueron sometidos a tratamiento estadístico (SPSS 22). De igual manera, se aplicó a la misma muestra, una Escala de Adicción a Internet, se tabularon los resultados y se sometieron a tratamiento estadístico (SPSS 22), al igual que en el primer caso. Antes de la aplicación del material se procedió a aplicar el formulario de Consentimiento Informado en donde se solicitó a cada participante el uso de los datos para los fines de la investigación. Posteriormente se realizó el análisis de los resultados estadísticos con el fin de obtener las conclusiones.

\section{RESULTADOS}

Con respecto a la Escala de Procrastinación Académica del total de la población encuestada se obtuvo los siguientes resultados donde $35.12 \%$ presenta 
un nivel Alto de procrastinación académica y un $58.33 \%$ presenta un nivel Moderado de procrastinación académica.

Tabla 1

Niveles de procrastinación académica

\begin{tabular}{lcc}
\hline Niveles & \multicolumn{2}{c}{ Procrastinación Académica } \\
\cline { 2 - 3 } & $\mathrm{F}$ & $\%$ \\
\hline Bajo & 11 & $6.55 \%$ \\
Moderado & 98 & $58.33 \%$ \\
Alto & 59 & $35.12 \%$ \\
\hline
\end{tabular}

En cuanto a los resultados de los niveles de adicción a internet se halló que de la población encuestada el $11.91 \%$ representa un nivel Alto de adicción a internet y un $49.40 \%$ presentan un nivel Moderado de adicción a internet.

Tabla 2

Niveles de adicción a internet

\begin{tabular}{lcc}
\hline \multirow{2}{*}{ Niveles } & \multicolumn{2}{c}{ Adicción a Internet } \\
\cline { 2 - 3 } & $\mathrm{F}$ & $\%$ \\
\hline Bajo & 65 & $38.69 \%$ \\
Moderado & 83 & $49.40 \%$ \\
Alto & 20 & $11.91 \%$ \\
\hline
\end{tabular}

En los resultados obtenidos se ha encontrado que existe una relación positiva y significativa entre procrastinación académica y adicción a internet $(r=0.322 ; p<0.05)$ en la población encuestada; $y$ en relación al sexo se han encontrado diferencias significativas. Considerando los resultados con respecto al sexo masculino se ha demostrado que existe una relación significativa entre procrastinación y adicción $(r=0.353 ; p<0.05)$ a diferencia del sexo femenino $(r=0.205 ; p>0.05)$.

Adicionalmente, de acuerdo a los resultados se ha encontrado que existe una relación significativa entre los niveles de procrastinación académica y adicción a internet $\left(X^{2}=10,313 ; p<0.05\right)$ lo cual nos refiere una relación importante entre estas variables en la población encuestada.

\section{DISCUSIÓN}

De acuerdo a Steel (2007), la procrastinación académica se inicia en laeducación secundariay persiste en la universidad, donde más del 50\% de los estudiantes informan de retrasos constantes y problemáticos en la realización de sus tareas, corroborando los resultados obtenidos en nuestra investigación donde más de un tercio de la población encuestada posee un alto grado de procrastinación académica y más de la mitad de la población encuestada demuestra un grado moderado de procrastinación académica.

La primera dimensión de la evaluación de procrastinación mide la autorregulación académica, donde más de la mitad de estudiantes encuestados demuestran una falta de autorregulación a la hora de realizar o cumplir con sus compromisos académicos demostrando así un déficit en su autorregulación. Hsin y Nam (2005), señalan cómo diferentes investigaciones han determinado que las personas que procrastinan se caracterizan por presentar un déficit en autorregulación y una tendencia a aplazar, por falta de tiempo las actividades que les llevarán a alcanzar una meta; no obstante, la procrastinación no sólo implica una dificultad en el manejo de tiempo, sino que se constituye como un proceso complejo que involucra variables afectivas, cognitivas y de comportamiento, lo que se demuestra en nuestra investigación en donde más de la mitad de estudiantes refiere que, no estudia por adelantado, no invierte tiempo necesario en estudiar y no trata de terminar el trabajo con tiempo de sobra.

Asimismo su tendencia a aplazar sus actividades estaría vinculada a la segunda dimensión de la evaluación de procrastinación en la cual se mide la postergación de actividades, en donde un tercio de la población encuestada, deja la tarea para último momento, postergan los trabajos y postergan las lecturas de los cursos que no les gustan. En este sentido, de acuerdo a lo que manifiestan Hsin y Nam (2005), no solo se toma en cuenta el hecho de postergar o dejar para después las tareas que pueden ser desarrolladas al momento, sino que, se pierde la esencia de la importancia que tiene realizar tareas o actividades en 
forma planificada y ordenada, obteniendo así mayores beneficios y satisfacciones para la consecución de objetivos tanto a corto como a mediano plazo.

Goldberg (1995), propone un conjunto de criterios para diagnosticar la adición a internet, basándose en los criterios diagnósticos de la dependencia a sustancias. Uno de los factores que menciona consiste en los deseos, de uno o más intentos fallidos de controlar el uso de internet. En los resultados que hemos obtenido apreciamos que más de un tercio de la población encuestada señala que, a menudo o siempre ha intentado estar menos tiempo conectados a internet pero no han podido. Lo cual quiere decir que hay un intento por parte de los estudiantes por controlar el uso de internet pero sin éxito. Corroborando lo que refieren diversos autores en donde el uso continuo del internet los perjudica en diferentes ámbitos de su vida como el social, familiar y el académico pudiéndolos llevar al fracaso académico (Kraut, Kiesler, Mukhopadhyay, Scherlis \& Patterson, 1998; LaRose, Eastin \& Gregg, 2001; Suler, 2001; Young, Pistner, O”Mara \& Buchanan, 2000; citados por Astonitas, 2005).

Al analizar los resultados encontrados en la presente investigación, podemos encontrar que existe una relación positiva y significativa entre procrastinación académica y adicción a internet en la población encuestada. En este estudio podemos darnos cuenta, a la luz de los resultados encontrados, que más de la mitad de los estudiantes tienen problemas en la autorregulación de las conductas académicas lo cual se relaciona directamente con la falta en el control relacionado al uso de internet. Los estudiantes al no poder controlar el uso de internet pueden perjudicar el cumplimiento de las tareas y trabajos académicos ya que vemos como una conducta recurrente la postergación de actividades académicas en más de la mitad de la población encuestada.

Rivas (2006) citado por Quant y Sánchez (2012), señala que la procrastinación se presenta porque las personas optan por realizar actividades que tengan consecuencias positivas a corto plazo, en lugar de llevar a cabo a aquellas que impliquen consecuencias demoradas. Según Echeburúa y Corral (2010), las Tics simplifican considerablemente nuestros quehaceres cotidianos. El atractivo de internet para los jóvenes es que se caracteriza por la respuesta rápida, las recompensas inmediatas, la interactividad y las múltiples ventanas con diferentes actividades. Esto se puede apreciar en la presente investigación en donde tenemos como resultado que más de la mitad de los estudiantes universitarios encuestados le dedican más tiempo del que habían planeado al uso de internet. Vemos actualmente que el uso de internet aumenta en los jóvenes y, cómo este tiempo utilizado en internet, va en detrimento de las conductas de autorregulación académica y de responsabilidad académica.

Por último, se observa en los resultados que existe diferencia entre procrastinación académica y adicción a internet con respecto al sexo de los participantes. En el caso de los estudiantes de sexo masculino existe una relación significativa entre procrastinación académica y adicción a internet. Esto no sucede en el caso de las estudiantes de sexo femenino. En un estudio hecho por Matalinares et. al., (2013), concluyen que existen diferencias significativas en la adicción a internet según sexo, ya que la población de sexo masculino está predispuesta a desarrollar una adicción al Internet a diferencia del sexo femenino. Por otro lado afirman que un motivo significativo por el cual usan el internet es por la búsqueda de la privacidad, asociada a la búsqueda de la identidad personal en los adolescentes, y el interés social por interactuar con el grupo de pares. Dado que la masificación del uso de internet en los últimos años, ha generado un medio de comunicación, de interacción y de organización social diferente, sobre la que se basa una forma nueva de sociedad. Resaltan que más del $70 \%$ de los encuestados de sexo masculino hace uso de internet para jugar en red, es por esta razón que puntualizan que estudios recientes demuestran que los individuos con riesgo de adicción a internet tiene niveles de adicción a los juegos en línea, adicción al cibersexo, ideación suicida y al abuso del alcohol, esto afirmado por Tsouvelas y Giotakos (2011) (citado en Matalinares et. al., 2013, p. 88).

El día de hoy, dados los resultados encontrados en la presente investigación, nos cuestionamos sobre las consecuencias de un uso desmedido de las tecnologías de información en las nuevas generaciones. Preguntarnos cómo podemos regular esta conducta para poder evitar la adicción a internet cuando vemos que los adolescentes el día de hoy utilizan sus celulares 
durante la mayor parte del día, cuando están en una reunión con su grupo de pares, cuando almuerzan con su grupo familiar o amigos. Con solo observar nuestro entorno, no solo podemos darnos cuenta de las diferencias generacionales que existen, sino, que son generaciones nacidas en una era digital. Asimismo, de qué manera poder distinguir entre una adicción a internet y el uso propio de la tecnología con la que esta generación ha estado inmersa desde su nacimiento, constituyendo en ellos nuevas características, como; el recibir de forma ágil e inmediata la información, ser atraídos por multitareas y procesos paralelos, teniendo un mejor funcionamiento y rendimiento cuando trabajan en red, prefiriendo instruirse de forma lúdica en lugar de la tradicional, entre otras. Por otro lado, la procrastinación académica se manifiesta desde mucho tiempo atrás, pero dado los adelantos en la tecnología en la actualidad podemos ver cómo ésta puede influir en incrementar o disminuir la conducta procrastinadora. Los dispositivos como los celulares, tablets, iPod, etc., los tenemos a la mano el día de hoy, y la velocidad en las comunicaciones, el online, se ha incrementado de manera vertiginosa. Sería interesante para una futura investigación el poder determinar en qué medida el uso de internet contribuye a esta conducta a lo largo del tiempo con un estudio longitudinal. Por estos motivos vemos la importancia de continuar las investigaciones en este tema para poder determinar las consecuencias de un uso inadecuado de la tecnología y poder tomar medidas preventivas.

\section{RECOMENDACIONES}

Es necesario contar con actividades preventivo promocionales, con el fin de ofrecer herramientas necesarias para lograr un oportuno y eficaz desempeño universitario. Tanto en la administración del tiempo como en el uso efectivo y benéfico del internet.

Académicamente desarrollar estrategias pedagógicas que les permitan a los estudiantes universitariosmodificarlasconductasdeautorregulación y postergación de actividades académicas, resaltando la importancia en el cumplimiento de las actividades y tareas encomendadas.

Generar en los estudiantes un mayor control en el uso efectivo del internet, que puedan aprovecharlo como un fin eficaz para el logro de sus objetivos y brindarles herramientas con la finalidad de lograr capacidades para hacer frente a las obligaciones académicas utilizando el internet como una herramienta más para su óptimo desarrollo.

El objetivo terapéutico en la adicción a internet es el reaprendizaje de la conducta de una forma controlada y desarrollando estrategias que le permitan un uso adecuado y dirigido por objetivos específicos que reafirmen su autorregulación y autocontrol.

\section{REFERENCIAS}

Álvarez, Ó. (2010). Procrastinación general y académica en una muestra de estudiantes de secundaria de Lima Metropolitana. Persona 13, pp. 159-177.

Angarita, L. (2012). Aproximación a un concepto actualizado de la procrastinación. Revista iberoamericana de psicología: ciencia y tecnología, 5 (2), pp. 85-94.

Astonitas,L. (2005). Personalidad, hábitos de consumo y riesgo de adicción al Internet en estudiantes universitarios. Revista de Psicología, XXIII, pp. $65-112$

Castellana, M., Sánchez-Carbonell, X., Graner, C. y Beranuy, M. (2007). El adolescente ante las tecnologías de la información y la comunicación. Internet, móvil y videojuegos. Papeles del Psicólogo, 28, pp. 196-200.

Chan, L. (2011). Procrastinación académica como predictor en el rendimiento académico en jóvenes de educación superior. Unife. Temát. Psicológica, 7(1), pp. 53-62.

Cía A. (2013). Las adicciones no relacionadas a sustancias (DSM-5, APA, 2013): un primer paso hacia la inclusión de las Adicciones Conductuales en las clasificaciones categoriales vigentes. Rev. Neuropsiquiatría 76 (4), pp. 210-217.

Echeburúa, E. y Corral, P. (2010). Adicciones a las nuevas tecnologías y a las redes sociales en jóvenes: un nuevo reto. Revista adicciones, 22(2), pp. 91-95. 
Goldberg, I. (1995). Internet addiction disorder (IAD). Diagnostic criteria. http://www.iuef.indiana.edu/ brown/hyplan/addict.htlm.

Hsin, A. \& Nam, J. (2005). Rethinking Procrastination: Positive effects of active procrastination behavior on attitudes and performance. The Journal of Social Psychology, 3, pp. 245-264.

Luengo, A. (2004). Adicción a Internet: conceptualización y propuesta de intervención. Revista Profesional Española de Terapia Cognitivo-Conductual 2, pp. 22-52.

Matalinares, M. et al, (2013). Adicción a la internet y Agresividad en estudiantes de secundaria del Perú. Revista IIPSI UNMSM, 16 (1), pp. 75-93.

Natividad, L. (2014). Análisis de la procrastinación en estudiantes universitarios. Tesis doctoral. Universidad de Valencia, España.
Oliva A., Hidalgo M., Moreno C., Jiménez L., Jiménez A., Antolín L., Ramos P. (2012). Trabajo de investigación: Uso y riesgo de adicciones a las nuevas tecnologías entre adolescentes y jóvenes andaluces. Departamento de Psicología Evolutiva y de la Educación Universidad de Sevilla.

Quant, D. \& Sánchez A. (2012). Procrastinación, procrastinación académica: concepto e implicaciones. Revista Vanguardia Psicológica Clínica Teórica y Práctica, 3(1), pp. 45-59.

Steel, P. (2007). The Nature of Procrastination: A MetaAnalytic and Theoretical Review of Quintessential Self-Regulatory Failure. Psychological Bulletin, 133(1), pp. 65-94.

Toro, J. (2010). El adolescente en su mundo. Riesgos, problemas y Trastornos. Madrid: Pirámide.

Fecha de recepción: 30 de octubre, 2017

Fecha de aceptación: 16 de noviembre, 2017 